\title{
Plantar Foot Interosseus Muscle
}

National Cancer Institute

\section{Source}

National Cancer Institute. Plantar Foot Interosseus Muscle. NCI Thesaurus. Code C52702.

Three small muscles located on the medial aspect of the third, fourth, and fifth metatarsal bones that facilitate adduction of the toes. 